\title{
Modulation of electron transport during Swing ECCD discharges in TCV
}

\author{
C. Zucca, O. Sauter, S. Alberti, S. Cirant ${ }^{1}$, T. P. Goodman, G. Turri \\ Centre de Recherches en Physique des Plasmas, \\ Association EURATOM-Confédération Suisse, EPFL, CH-1015 Lausanne, Switzerland \\ ${ }^{1}$ Istituto di Fisica del Plasma, EURATOM - ENEA - CNR Association, Milano, Italy
}

Abstract. Generation of a Swing Electron Cyclotron Current Drive (Swing ECCD), i.e. driving alternated, symmetric, positive or negative local ECCD, during a single discharge and at constant total input EC power, was performed at the Tokamak à Configuration Variable (TCV). The electron temperature is observed to be modulated inside the deposition radius, implying modulation of the electron transport properties. The modulation of ECCD is the only actuator for the observed modifications in the electron transport properties. These exhibit inverted behaviors depending on the deposition location of the co- and counter-ECCD. At more on-axis depositions, Swing ECCD results in a higher electron temperature during the co-ECCD phase, whereas at more off-axis depositions, the electron temperature is higher during the counter-ECCD phase. Transport modeling of these discharges shows that the local electron tranport behavior depends on the value of the modulated magnetic shear. The results are transport model independent, confirming the robustness of the magnetic shear modeling, and indicating that the main contribution is due to the ECCD. Moreover, the results are consistent with predictions from gyrokinetic simulations, that the local electron confinement is proportional to the magnetic shear at low shear, and inversely at high shear values, $s \gtrsim 1$.

\section{Introduction}

Understanding the physical connection behind the electron confinement and the plasma current density profile has become of primary importance for the most successful exploitation of a future fusion reactor. A precise understanding of transport, based on the experimental study of plasmas and on their modeling, allows one to identify the key parameters controlling the transport level and to predict the energy confinement time of future tokamaks, like the International Thermonuclear Experimental Reactor [1], [2]. The electron confinement and the current density profile were found to be strongly related by a variety of dedicated experiments [3], [4], [5] and theoretical investigations [6], [7]. The electron temperature behavior and the transport modeling of plasma discharges have been widely investigated in the Tokamak à Configuration Variable (TCV) [8], [9]. The current density profile being a key element which can strongly affect plasma turbulence, it is useful to design dedicated experiments specifically suitable for transport studies, solely based on the current profile modification. This work presents the analysis of TCV Swing ECCD discharges, which were designed with the purpose of realizing a modulation of the magnetic shear, in order to study the consequent effects on the central electron temperature (and thus of confinement properties). TCV Swing ECCD discharges are L-mode 
experiments, with modulation of ECCD at constant total input power [10], [11]. This allowed us to decouple the contributions of heating oscillations from those of current density tailoring. Therefore, any modifications in the transport properties of the plasma is to be ascribed only to the magnetic shear profile modulation realized by the ECCD swinging. The ECRH-ECCD system at TCV is equipped with two independently supplied clusters, each composed of 3 X2 gyrotrons, providing up to $3 \mathrm{MW}$ of total EC power. During a discharge, co- and counter-ECCD are alternatively driven at a constant frequency inside the plasma, maintaining a symmetric aiming of the EC beams, the same absolute value of total driven current, $I_{\text {eccd }}$, and the same EC power deposition profile, $P_{\mathrm{EC}}(\rho)$, where $\rho$ is a radial label describing the square root of the normalized volume: $\rho=\sqrt{V / V_{\text {tot }}}$ (indicated as $\rho_{\mathrm{V}}$ in the figures). This configuration is shown by the tokamak top view in Fig. 1. The target L-mode plasmas are characterized by the following range of parameters: plasma current $I_{\mathrm{p}}=120-150 \mathrm{kA}$, plasma elongation $\kappa \sim 1.2-1.4$, total input EC power $P_{\mathrm{EC}}=0.5-1 \mathrm{MW}$, line integrated electron density $n_{\mathrm{e} l}=1-1.510^{19} \mathrm{~m}^{-3}$, magnetic field at the axis $B_{0}=1.42 \mathrm{~T}$, modulation frequency $f_{\mathrm{ec}}=50 \mathrm{~Hz}$ (synchronous with the Thomson Scattering diagnostic). The first results [10] have been extended to study the radial dependence of electron transport in L-modes.

\section{Swing ECCD experiments}

Fig. 2 shows the time traces of some measured and reconstructed quantities during two Swing ECCD discharges (\#34064 and \#34044), realized at two different EC deposition radii, respectively $\rho_{\text {dep }}=0.3$ (Fig. 2, left) and $\rho_{\text {dep }}=0.55$ (Fig. 2, right). The time traces shown are: a) the constant plasma current $I_{\mathrm{p}}$ and, in arbitrary units, the modulated total EC power of the cluster of gyrotrons driving co-ECCD (the other cluster having a $180^{\circ}$ phase-shifted trace); b) the modulated central electron temperature, $\left.T_{\mathrm{e} 0} ; \mathrm{c}\right)$ the modulated line integrated electron density, $\left.n_{\mathrm{e} l} ; \mathrm{d}\right)$ the modulated internal inductance, $l_{\mathrm{i}}$; e) the modulated plasma elongation, $\kappa$. Due to the ECCD swing, $T_{\mathrm{e} 0}, n_{\mathrm{el}}, l_{\mathrm{i}}$ and $\kappa$ are modulated at the same modulation frequency. Fig. 3 shows the electron temperature, $T_{\mathrm{e}}$, and density, $n_{\mathrm{e}}$, profiles measured by Thomson Scattering, for each deposition. The profiles are obtained from the coherent time average over the final states of the two phases, the co- and counter-ECCD phases, for each discharge. For both discharges, the average over the co-ECCD phase includes the times: $t_{\mathrm{co}}=\left[\begin{array}{llllllll}0.5 & 0.7 & 0.9 & 1.1 & 1.3 & 1.5 & 1.7 & 1.9\end{array}\right] \mathrm{s}$, whereas the counter-ECCD phase includes: $t_{\text {counter }}=$ $\left[\begin{array}{lllllllll}0.6 & 0.8 & 1.0 & 1.2 & 1.4 & 1.6 & 1.8 & 2.0\end{array}\right]$ s. While the $n_{\mathrm{e}}$ profile stays approximately constant throughout the whole plasma evolution, the $T_{\mathrm{e}}$ profile appears modulated inside $\rho_{\text {dep }}$, at the same frequency of the EC alternate injection. At constant total EC power, this implies modulation of electron transport. The difference in $T_{\mathrm{e}}$ is not very large, but it is systematic. We tried to enhance the effect with increased EC power, but tearing modes were destabilized and modified too much the local transport properties, as discussed in Sec. 7. For the $\rho_{\text {dep }}=0.3$ deposition, the injection of co-ECCD results in a central $T_{\mathrm{e}}$ of about $2.05 \mathrm{keV}$, while injecting counter-ECCD decreases the value of central $T_{\mathrm{e}}$ to $1.75 \mathrm{keV}$. For the $\rho_{\text {dep }}=0.55$ deposition, the injection of co-ECCD results in a central $T_{\mathrm{e}}$ of about $1.2 \mathrm{keV}$, while 
injecting counter-ECCD increases the value of central $T_{\mathrm{e}}$ to $1.55 \mathrm{keV}$. Therefore, the experimental data show that the $T_{\mathrm{e}}$ modulations are opposite for the two depositions: in the more on-axis case, $T_{\mathrm{e} 0}$ is larger during the co-ECCD phase, with a $15 \%$ decrease from co- to counter-ECCD. On the other hand, for the off-axis case, $T_{\mathrm{e} 0}$ is larger during the counter-ECCD phase, with a $22 \%$ decrease from the counter- to co-ECCD phase.

A shot-to-shot scan in the deposition location has been performed in order to sweep across the radial region where the transport inversion occurs. In order to account for possible EC power misbalance and verify the reality of the phenomenon, all the new Swing ECCD discharges presented were realized in pairs, with the toroidal injection angle, $\phi$, of the two EC clusters inverted. The purpose of this procedure is to create pairs of Swing ECCD measurements in which the EC clusters driving co/counter-ECCD are flipped, while keeping all other actuators constant. In this way, the dominant phase observed does not depend on which gyrotron is used. Fig. 4 shows the ratio of a central Soft X-ray signal, averaged over the two separate phases, as a function of the deposition location, for the various Swing ECCD pairs realized, indicating that the global trend is maintained. Fig. 5 shows the time traces of three central X-ray measurements, for each of the two discharges discussed before as well as for their corresponding paired discharges. The pair of discharges reported in Fig. 5a was obtained with EC poloidal and toroidal injection angles $(\theta, \phi)=\left(120^{\circ}, \pm 17^{\circ}\right)$, corresponding to on-axis deposition $\left(\rho_{\text {dep }}=0.3\right)$, while the pair of discharges reported in Fig. 5b was obtained for $(\theta, \phi)=\left(106^{\circ}, \pm 24^{\circ}\right)$, corresponding to off-axis deposition $\left(\rho_{\mathrm{dep}}=0.55\right)$. The modulation is still observed in both discharges constituting the pair: for example, for the off-axis pair, the counter-ECCD phase, indicated by the red dots, is consistently higher than the co-ECCD phase, indicated by the blue dots, and for the on-axis pair the situation is inverted. The difference in signal intensities for each pair of discharges can be ascribed to small differences in heating between the two EC cluster gyrotrons and small differences in density.

\section{$3 \quad$ Modeling of Swing ECCD}

In order to link the inversion of transport properties to the magnetic shear, ASTRA modeling of the current density profile was performed. The numerical modeling allows to completely decouple the effects of the current profile modification from those of slight plasma heating misbalance or non-constant plasma elongation, which are key concepts at the basis of Swing ECCD and can be a rather delicate experimental issue. The ASTRA [12] transport code has been employed in both predictive and interpretative modes. For the predictive simulations, the Rebut-Lallia-Watkins (RLW, [13]) semi-empirical local transport model was used. In this model, the anomalous contribution of the electron heat diffusion coefficient is proportional to $q / s$. Two other predictive models, with different dependencies on the magnetic shear (quadratic and inversely quadratic), were also employed. These models are essentially a variation of the RLW model, in which the anomalous electron heat conductivity is prescribed by the RLW model multiplied by the cube or divided by the magnetic shear, respectively (see Appendix 
A). In the following analysis, they will be indicated as RLW $s^{3}$ and RLW/s. These modified models were used to compare the effect on $T_{\mathrm{e}}$ with a model having a different dependence of the electron heat diffusion coefficient on the magnetic shear. In the case of the interpretative analysis, the measured electron temperature profile, $T_{\mathrm{e}}(\rho, t)$, was prescribed as a time-varying input.

The quasi-linear CQL3D [14] Fokker-Planck code was used for the computation of the ECCD sources. The radial particle diffusion was taken into account (required due to the effect of fast particle transport, see [15]) as proportional to the electron heat diffusion. The methodology to determine $I_{\text {eccd }}$ requires a steady state, whereas Swing ECCD discharges are intrinsically in a transient state. An accurate determination of the value of the total EC current driven may only be obtained by increasing the period of the EC modulation, so as to reach a steady state during each of the co- and counter phases. Nevertheless, in order to have an estimate of the $j_{\text {eccd }}$ profile, the proportionality coefficient $D_{0}$ between the electron energy and particle coefficients has been fixed to a typical value used in various previous calculations of other plasma discharges [11], [8]: $D_{0}=0.2$ has been used for the two analyzed discharges. The $j_{\text {eccd }}$ profiles and corresponding values of $I_{\text {eccd }}$ calculated by CQL3D with $D_{0}=0.2$ are shown in Fig. 6 . The value of $I_{\text {eccd }}$, calculated by CQL3D with $D_{0}=0.2$, oscillates between $7.5 \mathrm{kA}$ and $-5.5 \mathrm{kA}$ on-axis, and between $5 \mathrm{kA}$ and $-5 \mathrm{kA}$ off-axis.

During a simulation, only few actuators were varied, such as the ECCD profile and the electron temperature profile (or equivalently the electron heat diffusion coefficient). All other parameters $\left(\kappa(t), P_{\mathrm{EC}}(\rho, t), n_{\mathrm{e}}(\rho, t), T_{\mathrm{i}}(\rho, t), I_{\mathrm{p}}(t)\right)$ were kept fixed. The numerical analysis was carried out for the TCV discharges of Fig. 2. The modulation period of these discharges is 200ms. Therefore, in the simulations the full co-ECCD and full counter-ECCD phases have been fixed to $95 \mathrm{~ms}$ each. The imposed time switch from one phase to the other is $5 \mathrm{~ms}$, of the order of the electron energy confinement time. The transition from one Swing ECCD phase to the other is imposed in such a way that the profiles of $j_{\text {eccd }}$ and $T_{\mathrm{e}}$ are varied in time following a linear interpolation between the co- and counterECCD profiles during 5ms; then, the profiles are held constant for 95ms. During this modulation period $(200 \mathrm{~ms})$, the plasma does not reach a full steady state, as the simulated parallel electric field is not yet completely relaxed. It would be interesting to extend the study of Swing ECCD discharges to steady state, too. However, the differences between the presented transient discharges and steady state ones are expected to be small. Simulations with much longer modulation times were also carried out, identifying the modulation period for steady state to be around $600 \mathrm{~ms}(300 \mathrm{~ms}+300 \mathrm{~ms})$.

\section{Interpretative simulation results}

For the interpretative simulations, the prescribed electron temperature is varied between the two profiles, measured by the Thomson Scattering system, shown in Fig. 3. The imposed electron temperature profile variation acts as a second actuator (along with $j_{\text {eccd }}$ ). Fig. 7 shows the calculated time traces of the various plasma current components and the prescribed, time-varying central $T_{\mathrm{e}}$, for the two analyzed discharges. As a consequence of the imposed modulation on $I_{\mathrm{eccd}}$ and $T_{\mathrm{e}}, I_{\mathrm{ohm}}$ is also modu- 
lated, whereas the modulation effect on $I_{\mathrm{bs}}$ is essentially negligible. Fig. 8 shows some simulated and imposed profiles of both discharges: the simulated magnetic shear (solid) and the imposed electron temperature profiles (dashed), in Fig. 8a and Fig. 8b; the safety factor (solid) and the EC power density profiles (dashed), in Fig. 8c and Fig. 8d; the simulated parallel electric field generated, in Fig. 8e and Fig. 8f.

At $\rho_{\text {dep }}=0.3$ (left column), with co-ECCD the value of $s$ is about 0.5 , whereas it increases to about 0.75 when counter-ECCD is injected. Therefore the reconstructed magnetic shear profile increases by $50 \%$ at the deposition location, when passing from the higher confinement co-ECCD to the lower confinement counter-ECCD phase; simultaneously, the central electron temperature is experimentally observed to drop by $15 \%$. The magnetic shear profiles are nevertheless modulated to the outside of the deposition region $(\rho>0.4)$, with a smaller and negative $\Delta s$, whereas they are not modified to the inside of it $(\rho<0.1)$. The values of the magnetic shear at the extremes of the deposition width are $s=0.1$ and $s=0.9$ (at $\rho=0.1$ and $\rho=0.4$, respectively).

At $\rho_{\text {dep }}=0.55$ (right column), with co-ECCD, the value of $s$ is about 0.7 , whereas it increases to about 1.1 when counter-ECCD is injected. Therefore an increment of $50 \%$ in $s$ at the deposition location is obtained for this off-axis case, when passing from the lower confinement co-ECCD to the higher confinement counter-ECCD phase. Contrary to the on-axis case, the central electron temperature is experimentally observed to increase by $25 \%$. The magnetic shear profiles are essentially not modulated, neither to the inside nor to the outside of the deposition width. The values of the magnetic shear at the extremes of the deposition width are $s=0.7$ and $s=1.7$, for $\rho_{\text {dep }}=0.55$ (at $\rho=0.4$ and $\rho=0.8$ respectively).

These simulations do not take into account a model for the sawtooth crash, as sawteeth are not experimentally observed in the analyzed discharges. As shown in Fig. 8c and Fig. 8d, the simulated $q=1$ surface, when occurring, is inside $\rho=0.1$ therefore if a sawtooth crash did occur, the mixing radius would be very small and should not affect the other reconstructed profiles in the deposition regions.

The time evolution of the parallel electric field profile, $E_{\|}$, is also shown, simulated during the switch from co- to counter-ECCD phase. The profiles are plotted every 10ms, with the blue and red profiles being the first and last respectively (color online). A large and rapid variation of the local $E_{\|}$is observed near the location of the deposition radius, the main change occurring during the first $20 \mathrm{~ms}$ following the imposed flip in ECCD sign. The initial and final profiles are not completely relaxed (flat) yet, therefore a steady state is not reached within 100ms. Finally, Fig. 9 shows the measured and simulated edge loop voltages of the on-axis deposition (the off-axis case being analogous). The experimental value of $Z_{\text {eff }}=3$ has been used in the modeling presented, and the agreement of the measured and reconstructed $V_{\text {loop }}$ is still maintained if the value of $Z_{\text {eff }}$ is increased to 3.5. It should be noted that all simulations described in this work were carried out by fixing the value of the plasma current $I_{\mathrm{p}}$, and modifying $V_{\text {loop }}$ according to the other calculated and measured quantities. 


\section{$5 \quad$ Predictive simulation results}

In the case of predictive simulations, $\chi_{\mathrm{e}}$ is calculated based on the RLW (or a modified RLW) model, whose explicit dependence in $s$ is detailed in Appendix A. We start from $\chi_{\mathrm{e}}=\chi_{\mathrm{e} L W}$ because the RLW scaling matches well TCV ohmic L-mode discharges [16]. The principal difference with respect to the interpretative cases is that now an imposed change in the current density profile (due to the Swing ECCD) will also affect the $s$-dependent $\chi_{\mathrm{e}}$ profile. This, in turn, will change the conductivity and bootstrap coefficients, therefore influencing the current density profile. With the difference in confinement properties observed for the two depositions taken into account, the usual RLW fails to reproduce the observed electron temperature trend on-axis (i.e. $T_{\mathrm{e}}{ }^{\text {co }}>T_{\mathrm{e}}{ }^{\text {counter }}$ ), because of its $1 / s$ dependence, therefore corrections to the RLW model were used for the separate discharges analyzed (see Appendix A).

Fig. 10 shows the calculated time traces of the various plasma current components and the calculated central $T_{\mathrm{e}}$, obtained with the $\chi_{\mathrm{e}, \mathrm{RLW} \cdot \mathrm{s}^{3}}$ (Fig. 10 left) and the $\chi_{\mathrm{e}, \mathrm{RLW} / \mathrm{s}}$ (Fig. 10 right) models, respectively for the on-axis and off-axis discharges. Different $s$ dependencies were tested in order to correctly simulate the $T_{\mathrm{e}}$ modulations, which are shifted by $180^{\circ}$ depending on the discharge analyzed. As a consequence of the imposed modulation on $I_{\mathrm{eccd}}$ and the $s$-dependent $\chi_{\mathrm{e}}, I_{\mathrm{ohm}}, T_{\mathrm{e} 0}$ and, to a minor extent, $I_{\mathrm{bs}}$ are also modulated.

Similar to what was found in the interpretative case, the simulated $s$ profiles are such that $s^{\text {counter }}>s^{\text {co }}$ around the deposition location, as shown in Fig. 11a and Fig. 11b. At $\rho_{\text {dep }}=0.3$, the values of the magnetic shear at the extremes of the deposition width are $s=0.1$ and $s=0.9$ (at $\rho=0.1$ and $\rho=0.4$, respectively). At $\rho_{\text {dep }}=0.55$, the values of the magnetic shear at the extremes of the deposition width are $s=0.4$ and $s=1.1$ (at $\rho=0.4$ and $\rho=0.8$, respectively). Therefore, the location and extent of the magnetic shear modulation around the deposition region are in agreement with the interpretative results. For both models, the maximum shear variation is $\Delta s=0.3$, occurring at the radial location of the EC deposition. Therefore, the conclusions on the modifications of the $q$ profile by Swing ECCD and on the time evolution of the magnetic shear are independent of the model. We have also verified that the effect of the experimental $T_{\mathrm{e}}$ profile does not affect the magnetic shear modulation simulated, as shown in Sec. 4.4.1 of Ref. [11].

The central $T_{\mathrm{e}}$ modulations follow the correct trend, since the models employed have an ad-hoc dependence on $s$. For the off-axis deposition, a stronger dependence in the magnetic shear, such as $\sim 1 / s^{3}$, may be required in order to obtain a difference in $T_{\mathrm{e}}$ at $\rho=0.4$ (just inside the $P_{\mathrm{EC}}$ deposition) of the same order as observed experimentally (Fig. 3b versus Fig. 11b).

The $q$ profiles are also shown in Fig. 11c and Fig. 11d, along with the EC deposition power, which is constant throughout all simulations. Fig. 11e and Fig. 11f show the time evolution of the parallel electric field profile, $E_{\|}$, is also shown, simulated during the switch from co- to counter-ECCD phase. 


\section{Agreement with previous simulations}

In the previous sections, it was shown that the simulated magnetic shear, around $\rho_{\mathrm{dep}}$, is consistently larger during the counter-ECCD phase. Since the transport behavior is inverted at different $\rho_{\mathrm{dep}}$, an increase of the confinement properties is observed if the magnetic shear decreases, in the radial region where the value of $s$ ranges from 0.1 to 0.9 (on-axis deposition). Likewise, a decrease of the confinement properties is observed if the magnetic shear decreases, in the radial region where the value of $s$ ranges from 0.7 to 1.7 (off-axis deposition). These results of the ASTRA modeling are in agreement with predictions from gyrokinetic linear simulations by Kinsey et al. [17], performed using the GYRO gyrokinetic code [18], with kinetic electrons. In these gyrokinetic simulations, the confinement properties increase if the magnetic shear decreases, in the radial region where $s<1$. At larger values of the magnetic shear (typically $s>1-1.5$ ), the opposite behavior is instead predicted (e. g. see Fig. 19 of Ref. [17], which shows the time-averaged ion and electron energy, and ion particle diffusivities as a function of $s$ ).

The interpretative, on-axis, time-varying $T_{\mathrm{e}}$ case moreover confirmed a previous modeling based on electrodynamics calculations, discussed in Ref. [10]. The main results arising from such modeling are that, around the deposition radius, $s$ passes from 0.2 to 0.7 , while switching from the co- to counterECCD phase, and $q\left(\rho_{\text {dep }}\right)$ oscillates between, respectively, 1.2 and 1.4. The $j_{\text {eccd }}$ profiles used in the previous modeling had been determined by the linear TORAY-GA code [19], while for the present calculations the co- and counter-ECCD profiles were calculated by solving the Fokker-Planck equation, including radial particle diffusion, as specified in Sec. 3. This results in ECCD profiles slightly broader than the ones predicted by linear theory. Nevertheless, the two models have shown similar relative changes in the magnetic shear modulation.

\section{MHD activity in Swing ECCD discharges}

Other Swing ECCD discharges were realized with larger EC power (i.e. $2+2$, or even $3+3$ gyrotrons) and feedback control on the plasma elongation. These were initially carried out with the purpose of creating a large database, featuring different values of the plasma current, radial deposition location, and width of the deposited power. Unfortunately, these discharges systematically exhibit MHD activity, therefore it is not easy to attribute the modulation of $T_{\mathrm{e}}$ uniquely to the magnetic shear oscillation. This obviously complicates the correct interpretation of the measured electron temperature modulation. Nevertheless, one can clearly observe that the mode activity is very intense during all co-ECCD phases and fades out when switching to counter-ECCD, as shown by the spectrogram of Fig. 12.

This confirms experimentally that the $q$ profile is, indeed, being modified locally, but may also explain the fact that no significant effect on $T_{\mathrm{e}}$ was observed in these discharges. However, the identification of the toroidal and poloidal mode numbers for these modes allows a validation of the ASTRA modeling by comparison with the simulated rational $q$ surfaces. For the discharge shown in Fig. 12 (\#30675), the mode was identified as $m / n=3 / 1$, located approximately in the region $0.4<\rho_{\psi} \lesssim 0.5$. Therefore 
an interpretative ASTRA simulation was carried out, in order to model the safety factor profile and identify the $q=3$ surface location. The model has employed ECCD profiles from CQL3D calculations, with $D_{0}=0.2$, according to which $I_{\text {eccd }}=13 \mathrm{kA}$, during the co-ECCD phase, and $I_{\text {eccd }}=-15 \mathrm{kA}$, during the counter-ECCD phase. Results from this simulation are shown in Fig. 13. The $q$ profile during the co-ECCD phase crosses $q=3$ at $\rho_{\psi} \sim 0.55$ (consistently with the mode location), whereas the surface is pushed outwards at $\rho_{\psi} \sim 0.7$ during the counter-ECCD phase. The tearing mode is responsible for the large difference observed in the $T_{\mathrm{e}}$ profiles and hence for the differences in the core $q$ profiles. Further analysis of the modes in these experiments is beyond the scope of the present study, but these modeled $q$ and $s$ profiles form an excellent basis for tearing mode studies and the effect of local current density profile perturbations.

\section{Conclusions}

The study on Swing ECCD has confirmed the link between the electron transport and the magnetic shear modulation. The ASTRA modeling has shown that the electron tranport behavior depends on the value of the modulated magnetic shear. The discharges presented are consistent with the inversion of the confinement properties, depending on the value of the magnetic shear, which is predicted by gyrokinetic simulations. Namely, the local electron diffusivity $\chi_{\mathrm{e}}$ is proportional to $s$ or $s^{2}$ for $s \sim 0-1$, while $\chi_{\mathrm{e}} \sim 1 / s$ or $\chi_{\mathrm{e}} \sim 1 / s^{2}$ for $s \gtrsim 1$. This is experimentally observed even when inverting the EC clusters toroidal angles, in order to flip systematic different heating effects. The location and extent of the magnetic shear modulation validate a previous, simpler model, based on electrodynamics calculations. In all, the numerical results carried out with ASTRA are transport-model-independent, therefore they confirm the robustness of the magnetic shear modeling and indicate that the main contribution is due to the ECCD modulation.

\section{A Transport models}

The semi-empirical RLW local transport model [13] has been validated to well reproduce TCV discharges [16]. It involves a critical electron temperature gradient: when the electron temperature gradient exceeds the critical value, a turbulent behavior in the magnetic topology is triggered. The electron heat transport coefficient consists of two contributions, a neoclassical and an anomalous one, therefore one can write: $\chi_{\mathrm{e}, \mathrm{RLW}}=\chi_{\mathrm{e}, \mathrm{neo}}+\chi_{\mathrm{e}, \mathrm{an}}$. The neoclassical contribution is evaluated using formulas given in [20]. The anomalous contribution is given by:

$$
\chi_{\mathrm{e}, \text { an }}=\frac{C_{\mathrm{e}, \text { an }}}{B_{\phi}} \sqrt{\frac{\epsilon\left(1+Z_{\mathrm{eff}}\right)}{R_{0}} \frac{T_{\mathrm{e}}}{T_{\mathrm{i}}}}\left|\left(\frac{\nabla T_{\mathrm{e}}}{T_{\mathrm{e}}}+2 \frac{\nabla n_{\mathrm{e}}}{n_{\mathrm{e}}}\right) \frac{q \rho}{s}\right|\left(1-\frac{\nabla T_{\mathrm{RLW}}}{\nabla T_{\mathrm{e}}}\right) H\left(\nabla T_{\mathrm{e}}-\nabla T_{\mathrm{RLW}}\right),
$$

where $B_{\phi}$ is the toroidal magnetic field, $\epsilon$ the inverse aspect ratio, $Z_{\text {eff }}$ the effective charge, $R_{0}$ the tokamak major radius, $T_{\mathrm{i}}$ the ion temperature, $H(x)$ the Heaviside step function, and the critical 
electron temperature gradient is given by the expression:

$$
\nabla T_{\mathrm{RLW}}=\frac{C_{\mathrm{gcrt}}}{q} \sqrt{\frac{\eta j B_{\phi}^{3}}{n_{\mathrm{e}} \sqrt{T_{\mathrm{e}}}}},
$$

$\eta$ being the neoclassical resistivity and $j$ the current density profile. Note that the critical value of $T_{\mathrm{e}}$ is essentially negligible outside the deposition region in case of ECH heated plasmas. In the RLW model, an increase on the local magnetic shear, with the safety factor relatively constant, induces a reduction of transport, $\chi_{\mathrm{e}, \text { an }} \sim|q / s|$, which is adequate for TCV discharges in case of negative or large positive magnetic shear. The two free parameters $C_{\mathrm{e} \text {,an }}$ and $C_{\mathrm{gcrt}}$ of the transport models were adjusted until they adequately reproduced the measured $T_{\mathrm{e}}$ for a pure ECH discharge, then subsequently held at those values during the simulations of the Swing ECCD discharges (for instance, $C_{\mathrm{e}, \text { an }}=0.6$ and $C_{\text {gcrt }}=1$ for the RLW model).

To test different dependencies on $s$, two modified versions of the RLW local transport model were also taken into account. Specifically:

$$
\begin{gathered}
\chi_{\mathrm{e}, \mathrm{RWL} \cdot \mathrm{s}^{3}}=\chi_{\mathrm{e}, \text { neo }}+\frac{C_{\mathrm{e}, \text { an }}}{B_{\phi}} \sqrt{\frac{\epsilon\left(1+Z_{\mathrm{eff}}\right)}{R_{0}} \frac{T_{\mathrm{e}}}{T_{\mathrm{i}}}}\left|\left(\frac{\nabla T_{\mathrm{e}}}{T_{\mathrm{e}}}+2 \frac{\nabla n_{\mathrm{e}}}{n_{\mathrm{e}}}\right) q \rho s^{2}\right|\left(1-\frac{\nabla T_{\mathrm{RLW}}}{\nabla T_{\mathrm{e}}}\right) H\left(\nabla T_{\mathrm{e}}-\nabla T_{\mathrm{RLW}}\right), \\
\chi_{\mathrm{e}, \mathrm{RLW} / \mathrm{s}}=\chi_{\mathrm{e}, \text { neo }}+\frac{C_{\mathrm{e}, \mathrm{an}}}{B_{\phi}} \sqrt{\frac{\epsilon\left(1+Z_{\mathrm{eff}}\right)}{R_{0}} \frac{T_{\mathrm{e}}}{T_{\mathrm{i}}}}\left|\left(\frac{\nabla T_{\mathrm{e}}}{T_{\mathrm{e}}}+2 \frac{\nabla n_{\mathrm{e}}}{n_{\mathrm{e}}}\right) \frac{q \rho}{s^{2}}\right|\left(1-\frac{\nabla T_{\mathrm{RLW}}}{\nabla T_{\mathrm{e}}}\right) H\left(\nabla T_{\mathrm{e}}-\nabla T_{\mathrm{RLW}}\right) .
\end{gathered}
$$



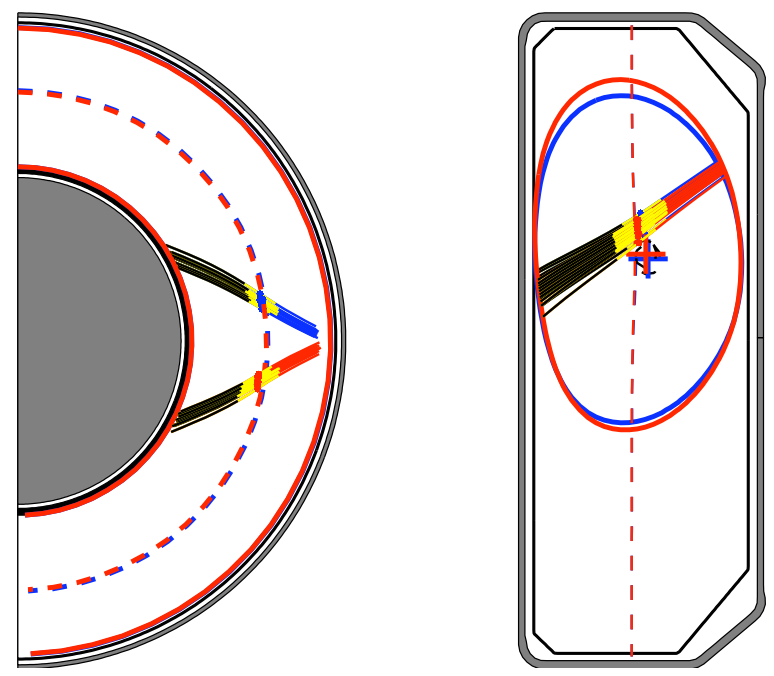

Figure 1: Top and side views of the EC beams injected in a typical Swing ECCD discharge. The blue and red color coded beams correspond, respectively, to the co- and counter-ECCD. The light gray section corresponds to 99\% absorption. The ray paths are calculated with the TORAY-GA ray-tracing code [19]. 

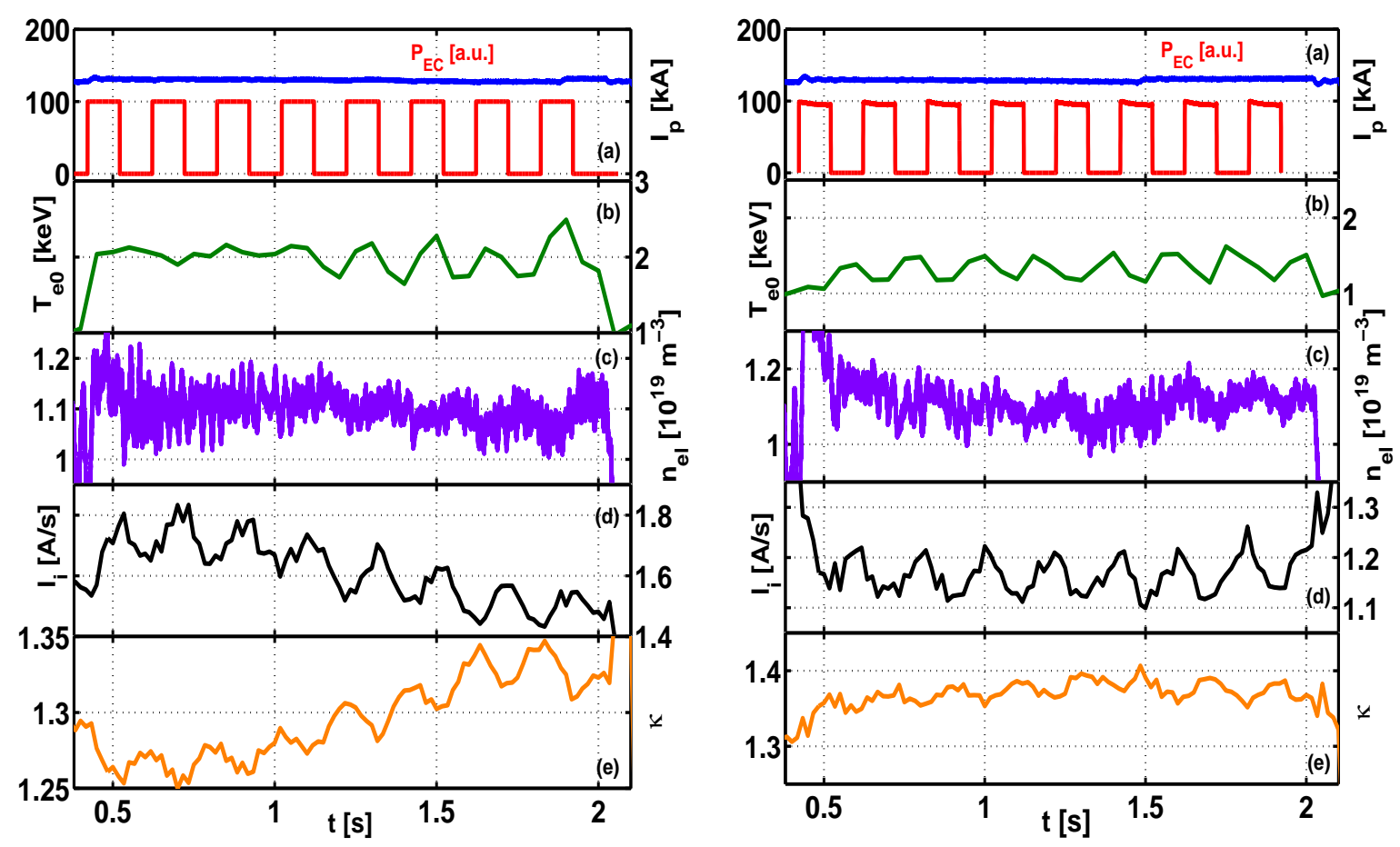

Figure 2: Time traces of two TCV Swing ECCD discharges (\#34064 and \#34044), in which the ECCD modulation is realized, respectively, at $\rho_{\mathrm{dep}}=0.3$ and $\rho_{\mathrm{dep}}=0.55$. a) Constant plasma current and modulated total input EC power for the cluster of gyrotrons driving co-ECCD. The EC power traces are in arbitrary units, and the values of the total injected $P_{\mathrm{EC}}$ are $450 \mathrm{~kW}$ for the on-axis discharge and $900 \mathrm{~kW}$ for the off-axis discharge. b) Modulated central electron temperature. c) Line integrated electron density. d) Internal inductance from the LIUQE reconstruction. e) Reconstructed plasma elongation. 

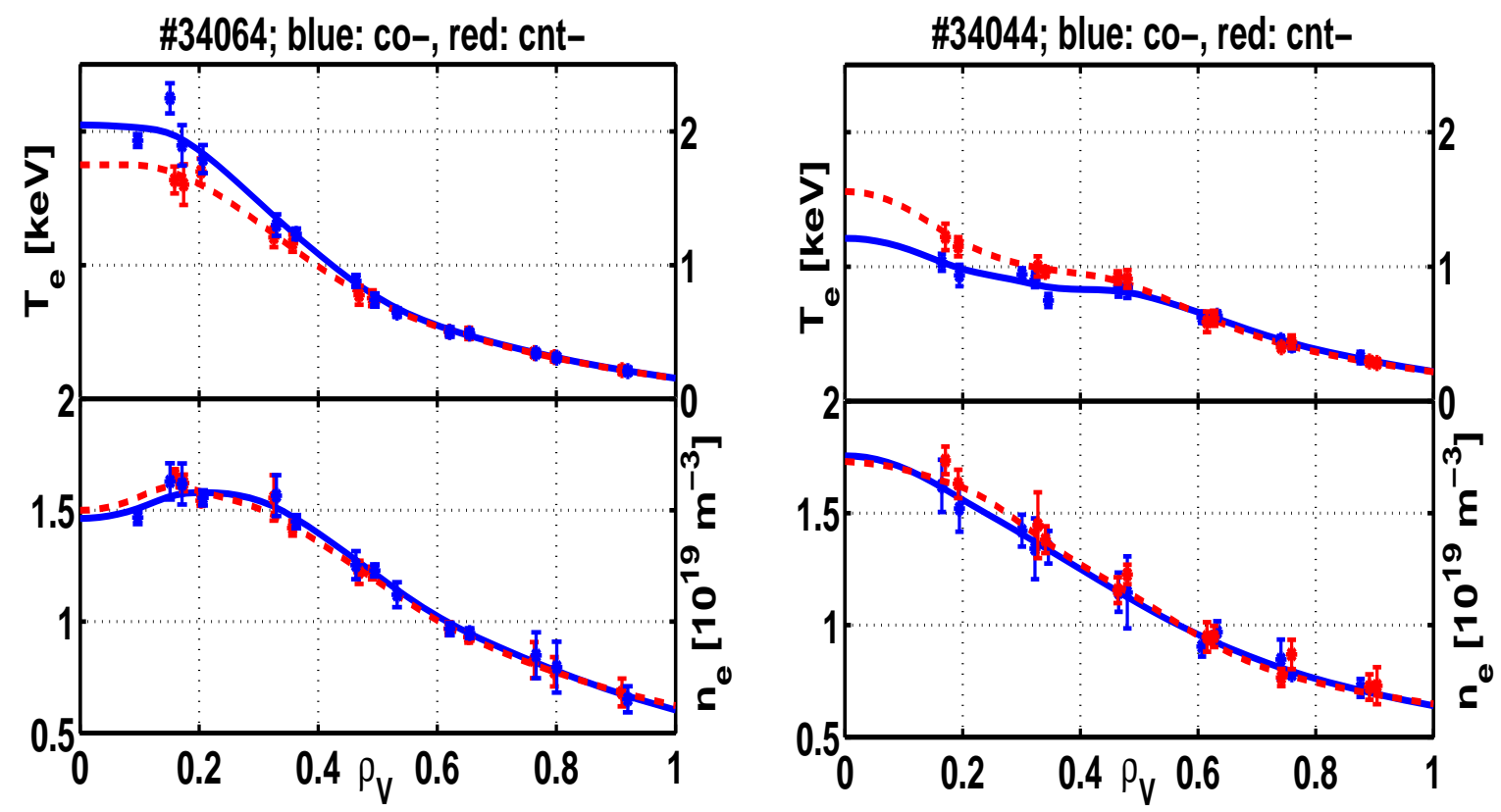

Figure 3: Radial profiles of the experimental Thomson Scattering $T_{\mathrm{e}}$ and $n_{\mathrm{e}}$ data, which are averaged over each phase and with bins of $\Delta \rho=0.05$, showing the modification of transport properties during the two Swing ECCD phases. For both discharges, the average over the co-ECCD phase includes $t_{\mathrm{co}}=\left[\begin{array}{llllllll}0.5 & 0.7 & 0.9 & 1.1 & 1.3 & 1.5 & 1.7 & 1.9\end{array}\right]$, whereas the counter-ECCD phase includes

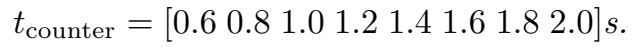




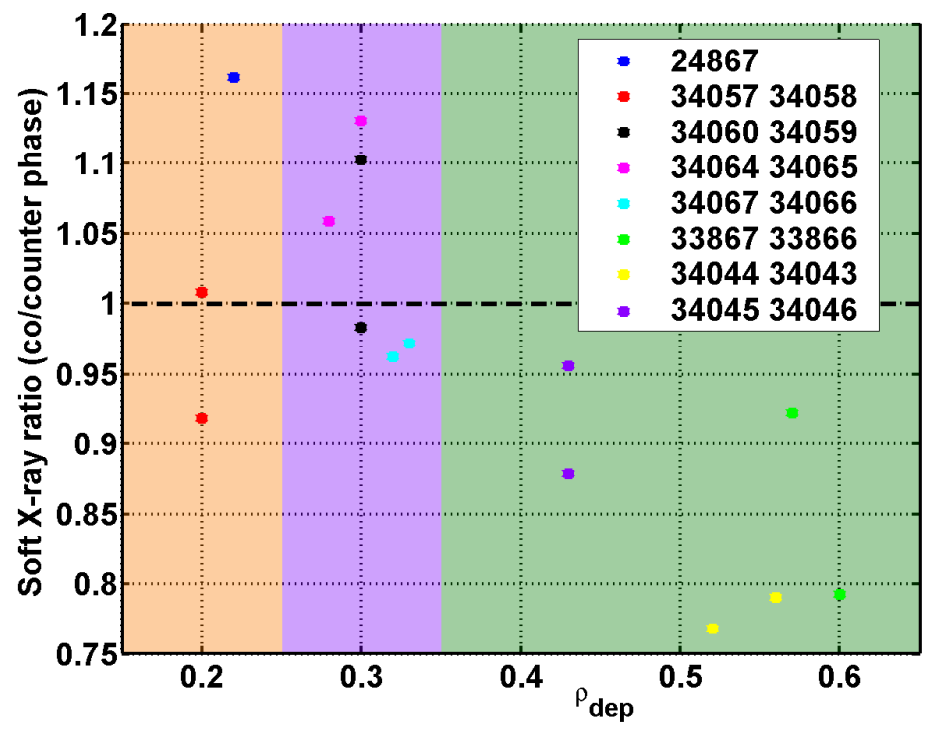

Figure 4: Ratio of the average Soft X-ray signal during the co-ECCD phase and the counter-ECCD phase for various Swing ECCD discharges, as a function of the deposition location (in $\rho$ ). 

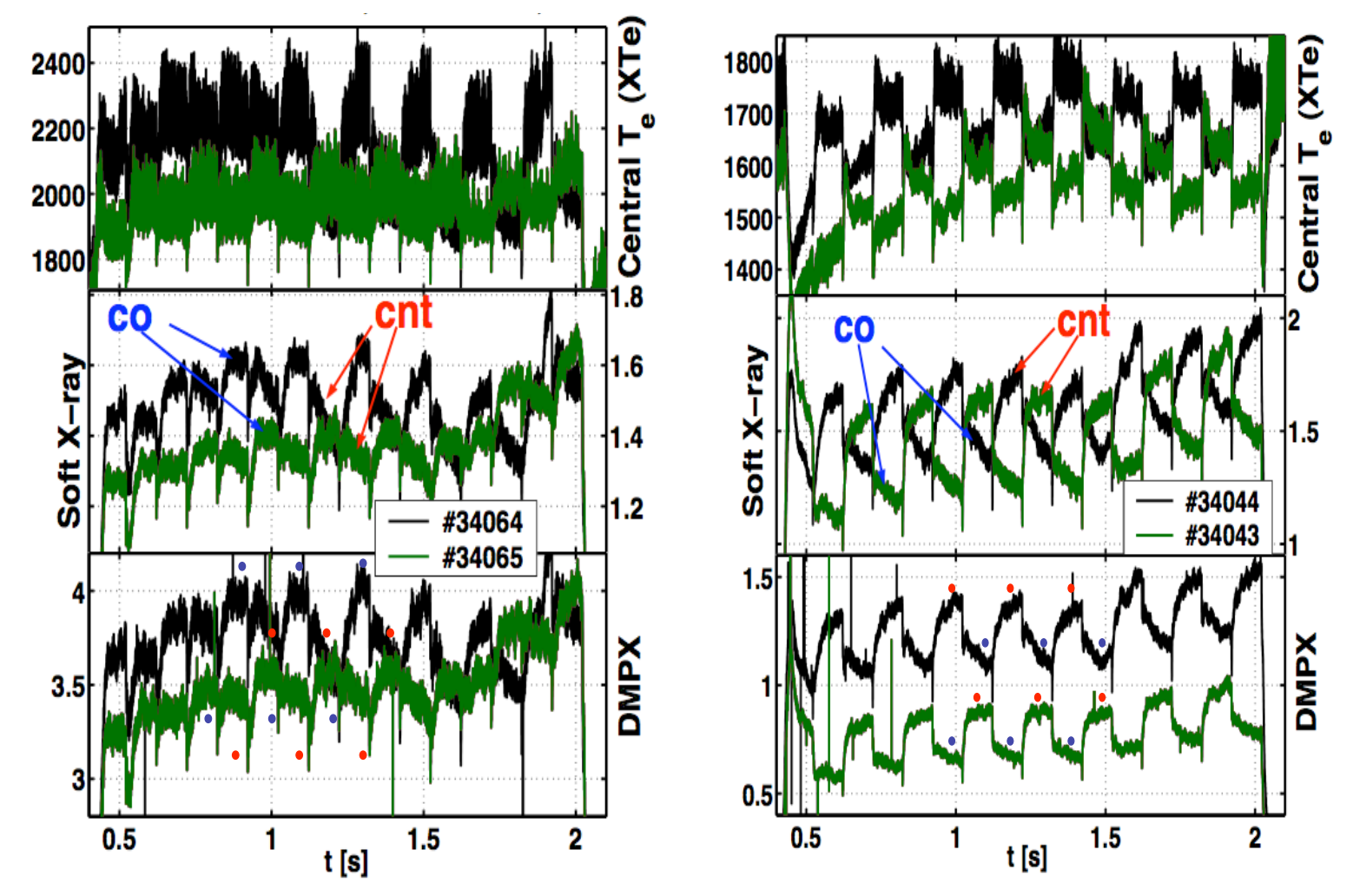

Figure 5: Time traces of three central X-ray measurements for two pairs of Swing ECCD discharges, with on-axis (left), and off-axis $\rho_{\text {dep }}$ (right). 


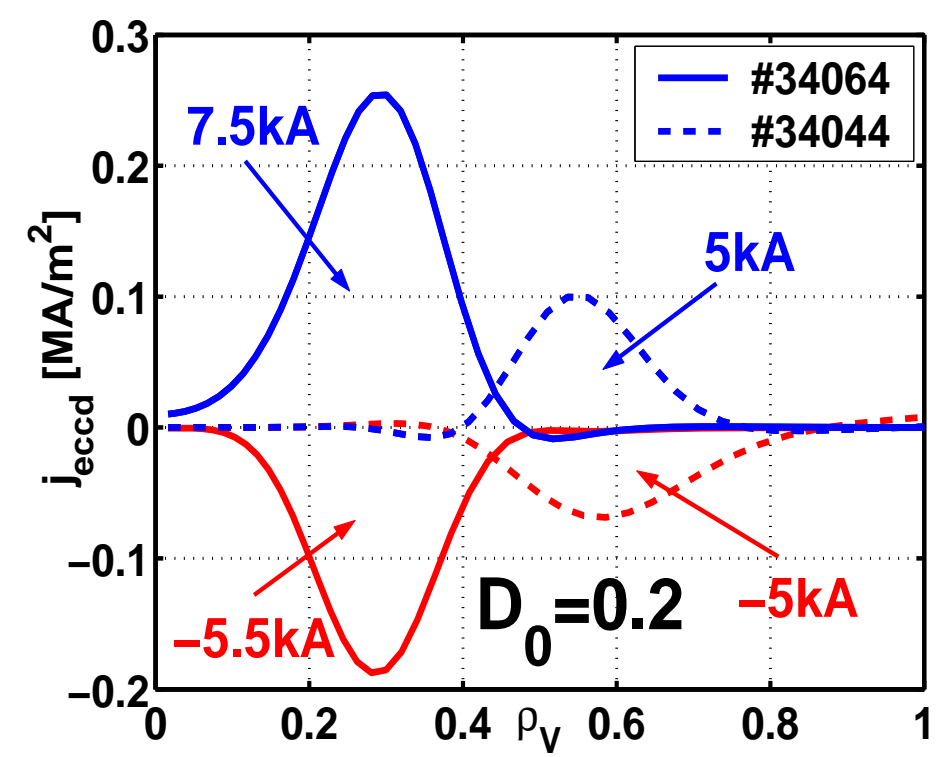

Figure 6: $j_{\text {eccd }}$ profiles (and corresponding values of $I_{\mathrm{eccd}}$ ) calculated by $C Q L 3 D$, assuming $D_{0}=0.2$. These profiles were used in all the following ASTRA simulations of Swing ECCD discharges. 


\section{\#34064}

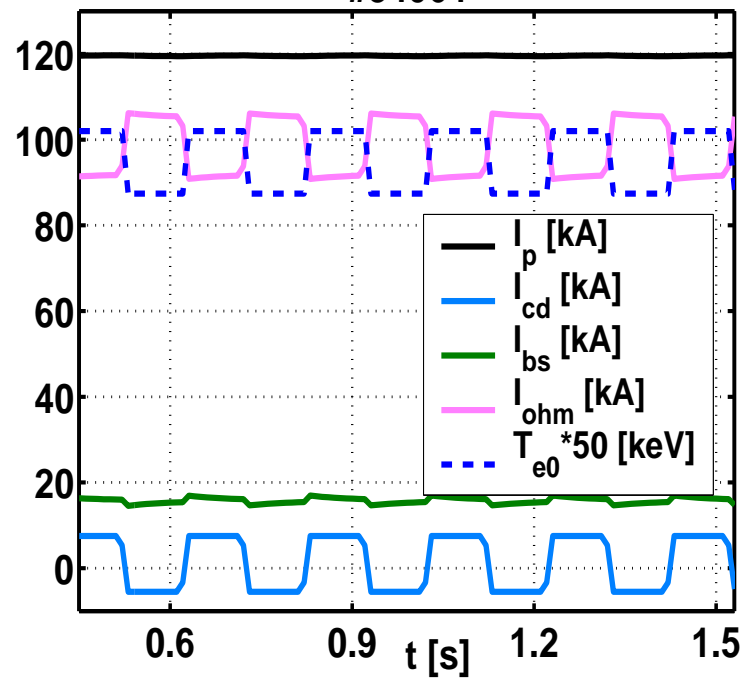

\#34044

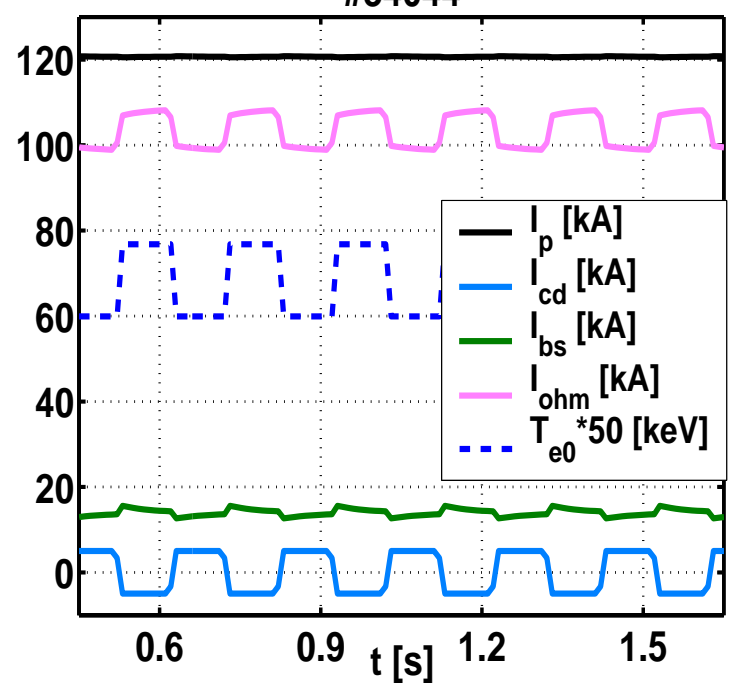

Figure 7: Simulated or imposed time traces of the various plasma current components and the imposed central $T_{\mathrm{e}}$, of the on-axis (a) and off-axis (b) deposition. In the ASTRA modeling, $I_{\mathrm{p}}, I_{\mathrm{cd}}\left(=I_{\mathrm{eccd}}\right)$ and $T_{\mathrm{e}}$ are imposed, whereas $I_{\mathrm{bs}}$ and $I_{\mathrm{ohm}}$ are calculated. Interpretative simulation results (color online). 


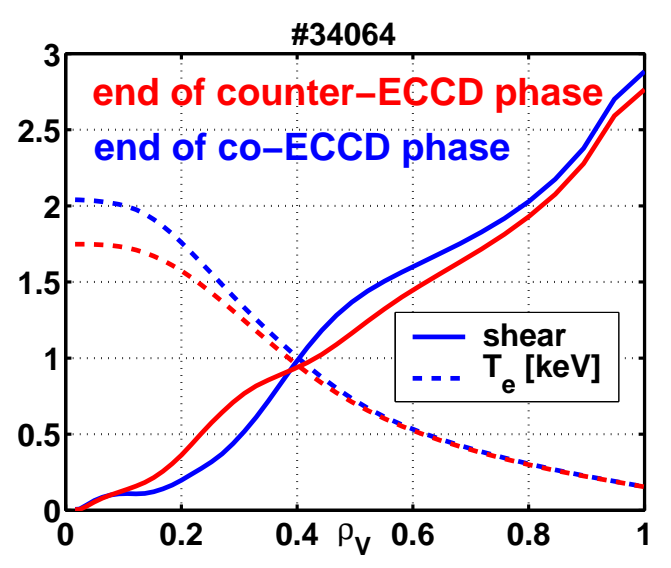

(a)

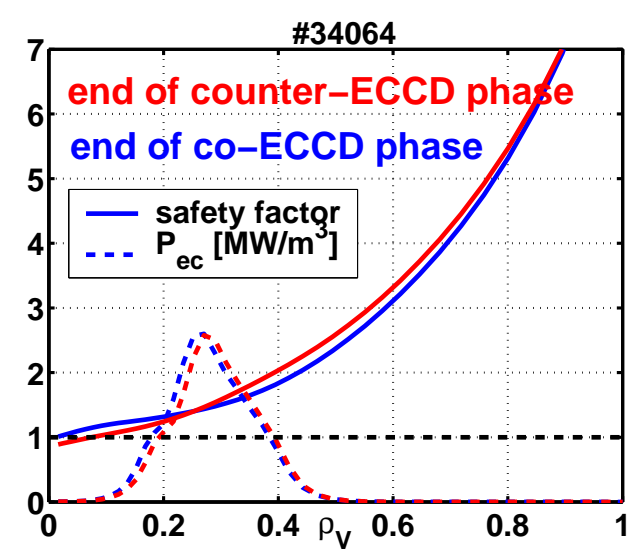

(c)

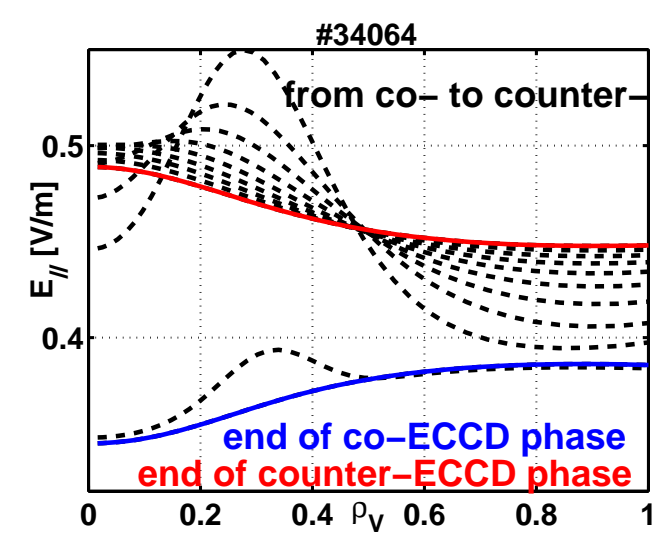

(e)

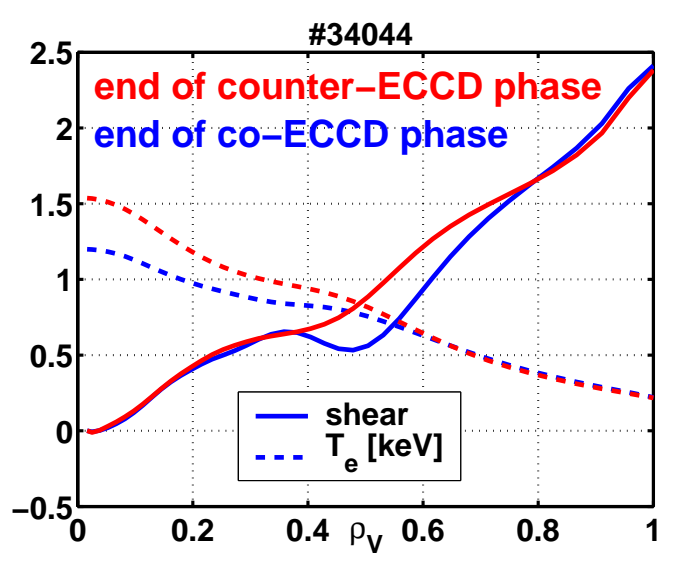

(b)

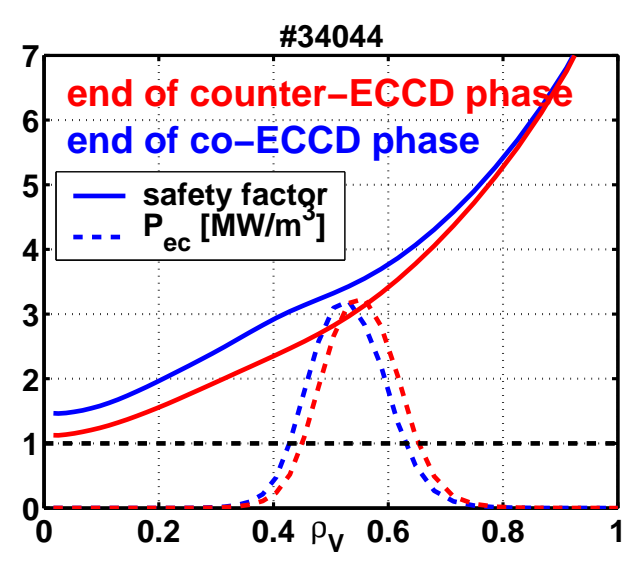

(d)

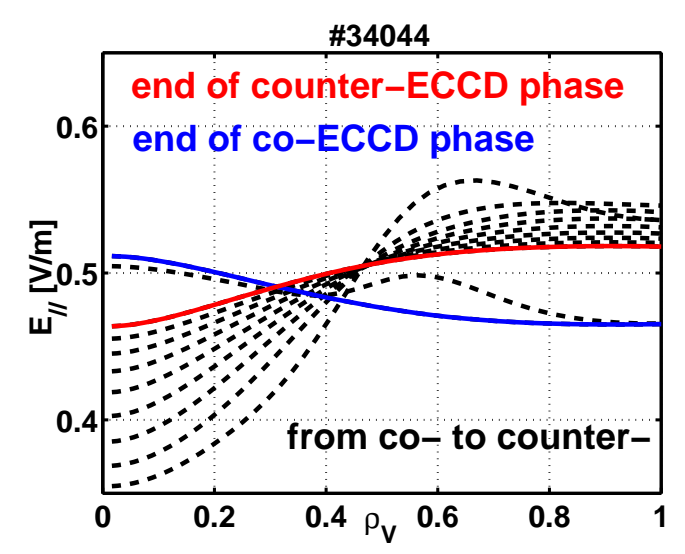

(f)

Figure 8: Simulated magnetic shear and imposed electron temperature profiles for the on-axis (a) and off-axis (b) deposition; simulated safety factor and deposited EC power density profiles for the on-axis (c) and off-axis (d) deposition; simulated parallel electric field profile for the on-axis (e) and off-axis (f) deposition. Interpretative simulation results (color online). 


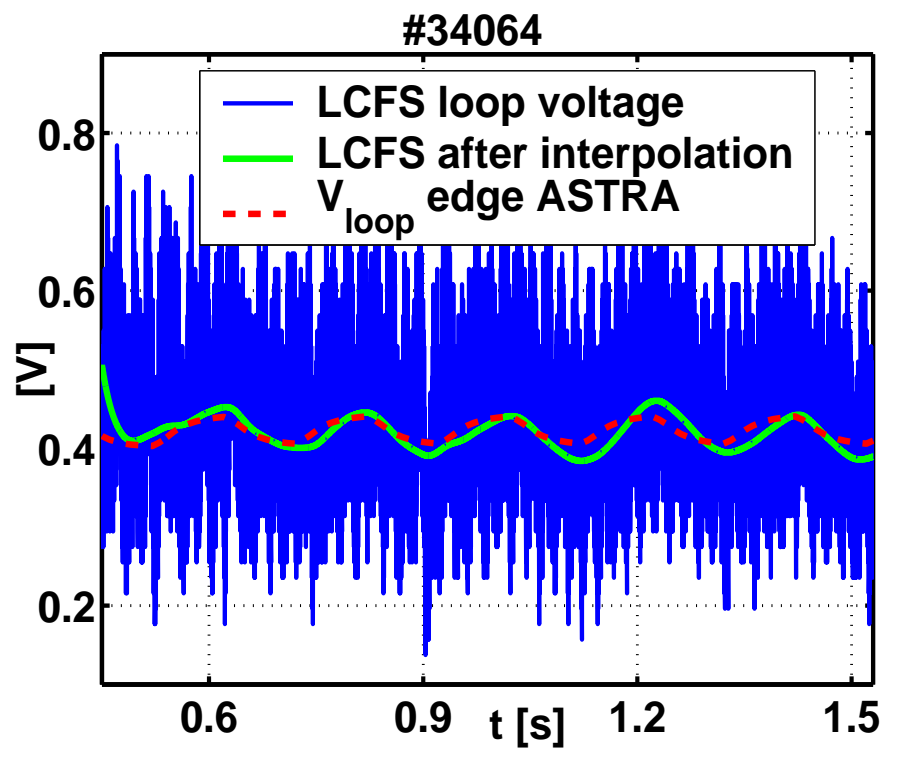

Figure 9: Time trace of the measured loop voltage (black noisy trace), its corresponding filtered signal by interpolation, and ASTRA simulated loop voltage (at constant $I_{\mathrm{p}}$ ) for the interpretative, on-axis case, and with $Z_{\text {eff }}=3$. The simulated $V_{\mathrm{loop}}$ is still in agreement with the experimental one if $Z_{\mathrm{eff}}$ is varied between 3 and 3.5 . 
\#34064

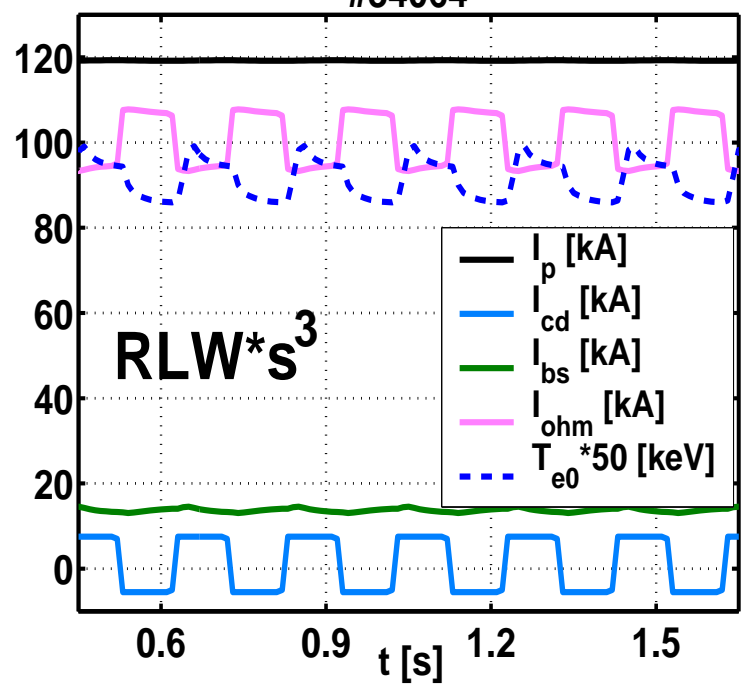

\#34044

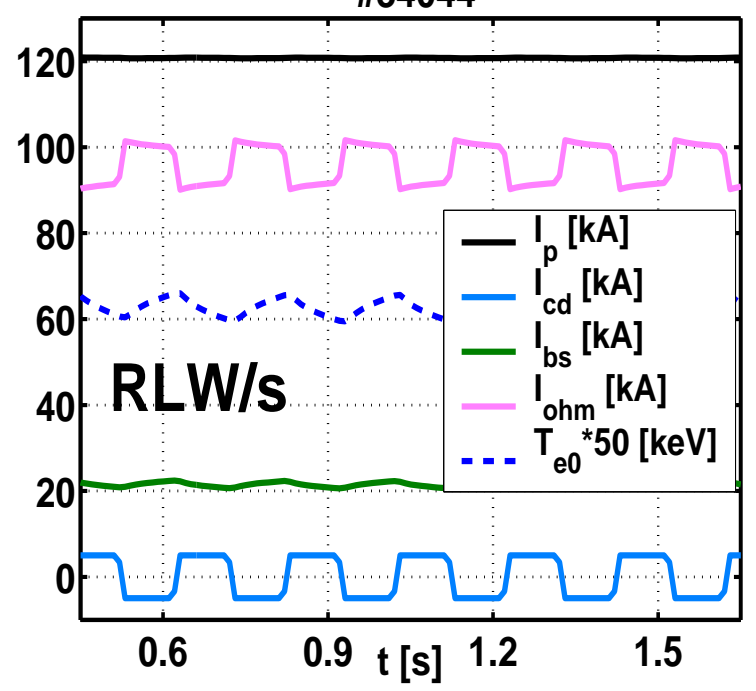

Figure 10: Simulated or imposed time traces of the various plasma current components and the simulated central $T_{\mathrm{e}}$, obtained in ASTRA with the modified $R L W \cdot s^{3}$ (left) and $R L W / s$ (right) models, respectively for the on-axis and off-axis deposition. In the ASTRA modeling, $I_{\mathrm{p}}$ and $I_{\mathrm{cd}}\left(=I_{\mathrm{eccd}}\right)$ are imposed, whereas $I_{\mathrm{bs}}, I_{\mathrm{ohm}}$ and $T_{\mathrm{e}}$ are calculated. Predictive simulation results (color online). 


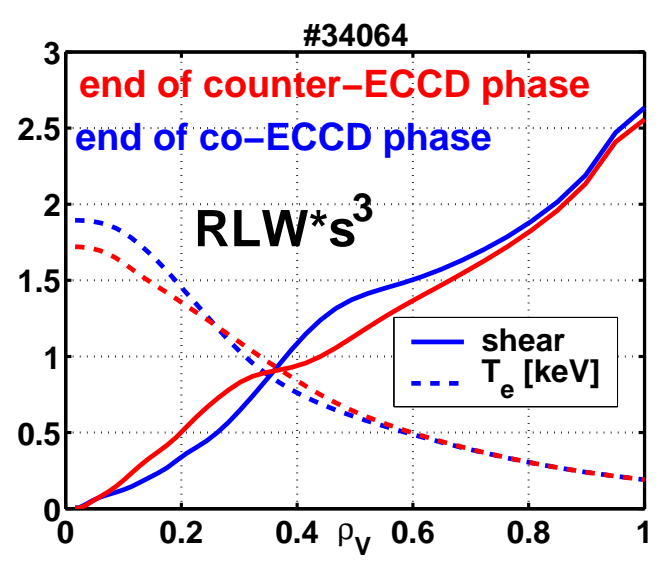

(a)

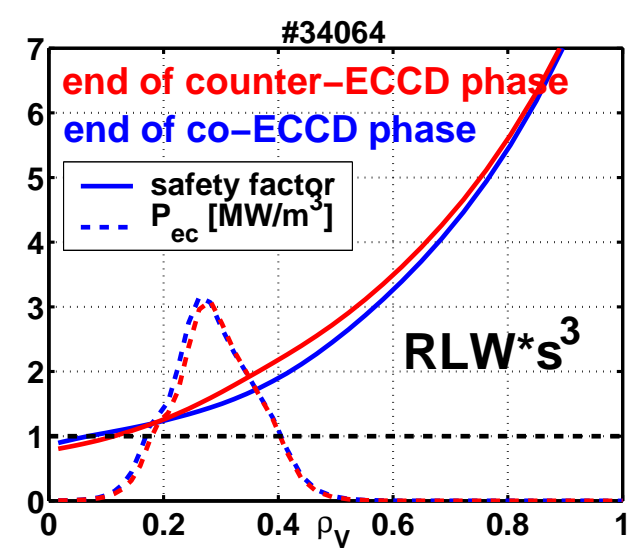

(c)

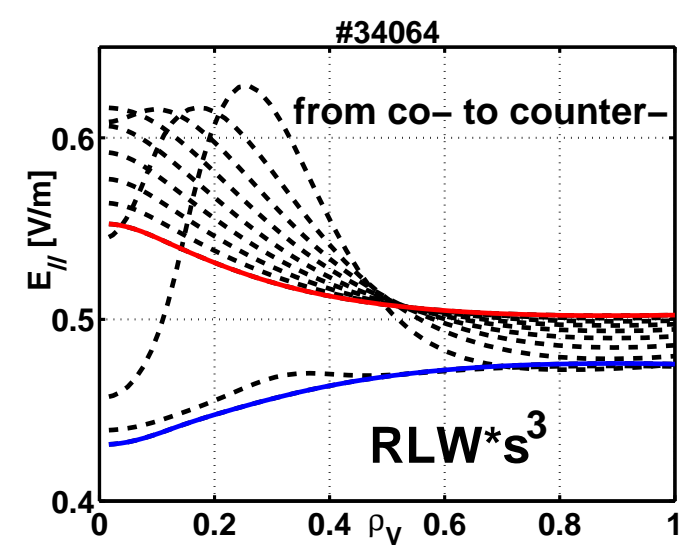

(e)

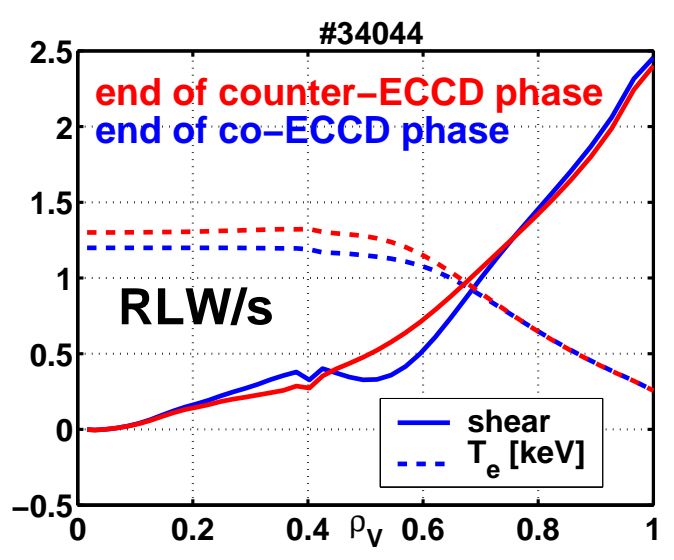

(b)

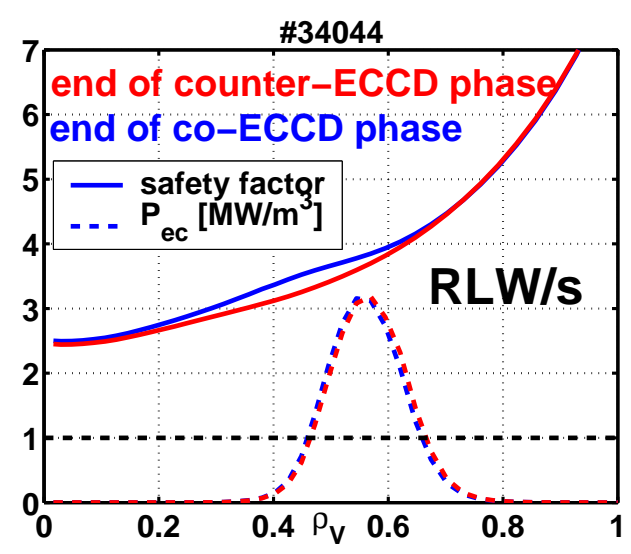

(d)

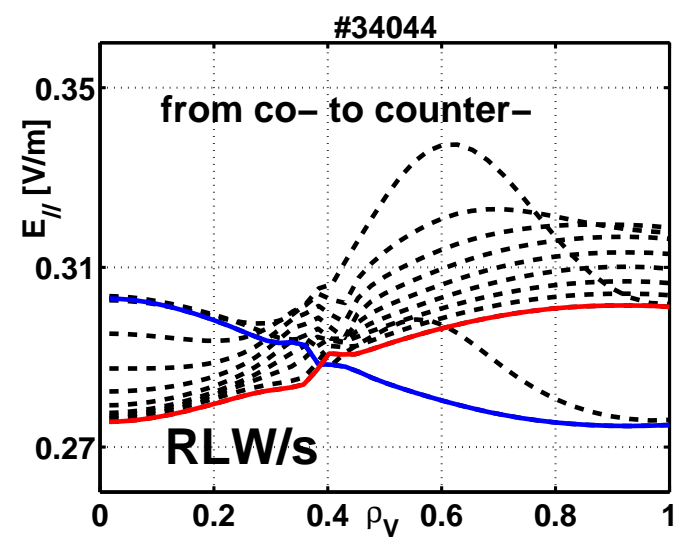

(f)

Figure 11: Simulated magnetic shear and electron temperature profiles for the on-axis (a) and off-axis (b) deposition; simulated safety factor and deposited EC power density profiles for the on-axis (c) and off-axis (d) deposition; simulated parallel electric field profile for the on-axis (e) and off-axis ( $f$ ) deposition. Predictive simulation results (color online). 


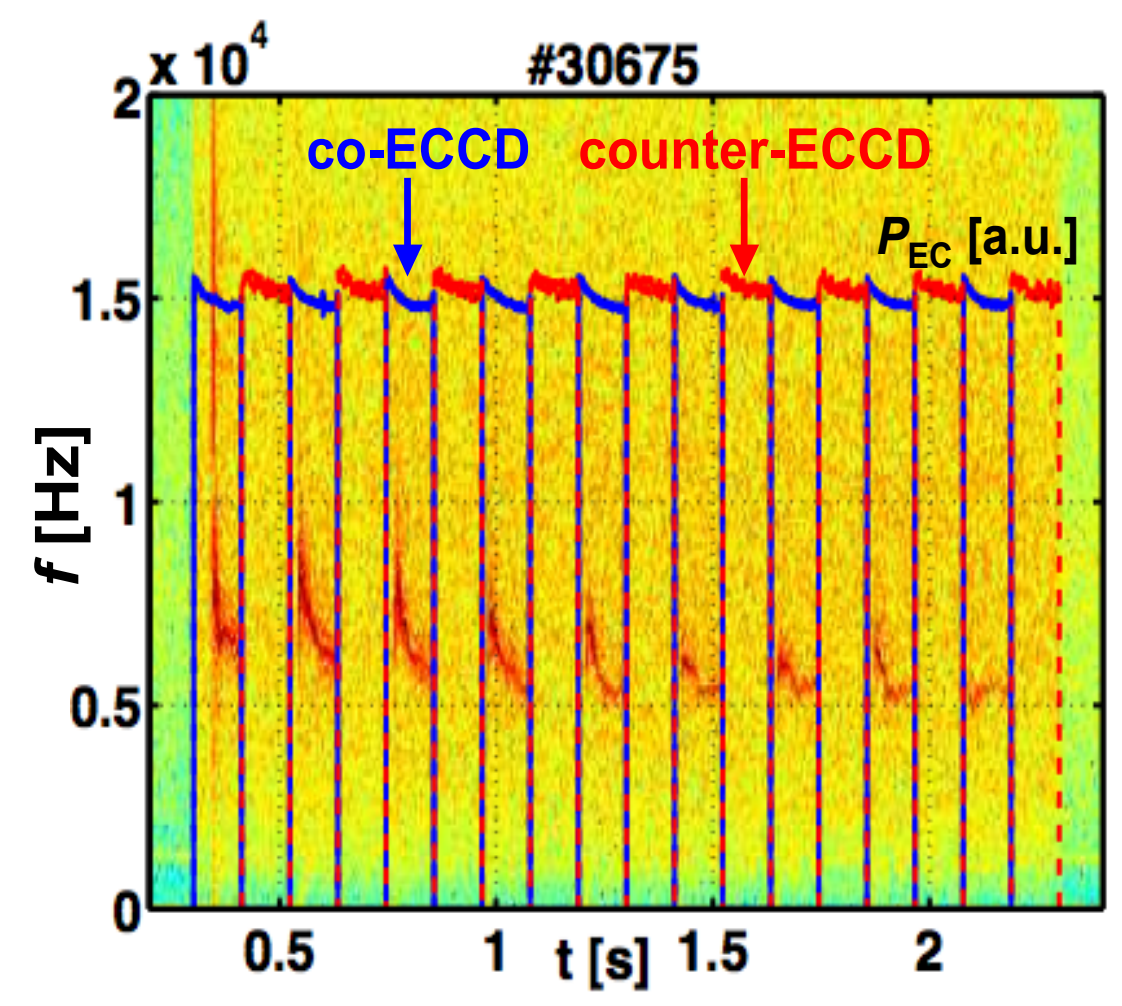

Figure 12: Spectrogram revealing the presence of MHD activity, with alternated phases of appearance and absence, for TCV discharge \#30675. The modulation frequency is $45 \mathrm{~Hz}$, and the EC total power is indicated in arbitrary units. 
\#30675

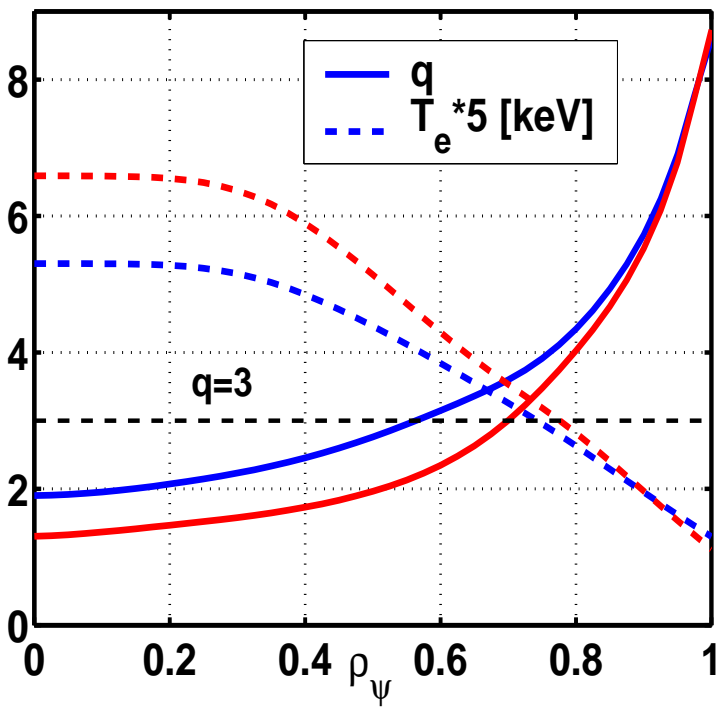

\#30675

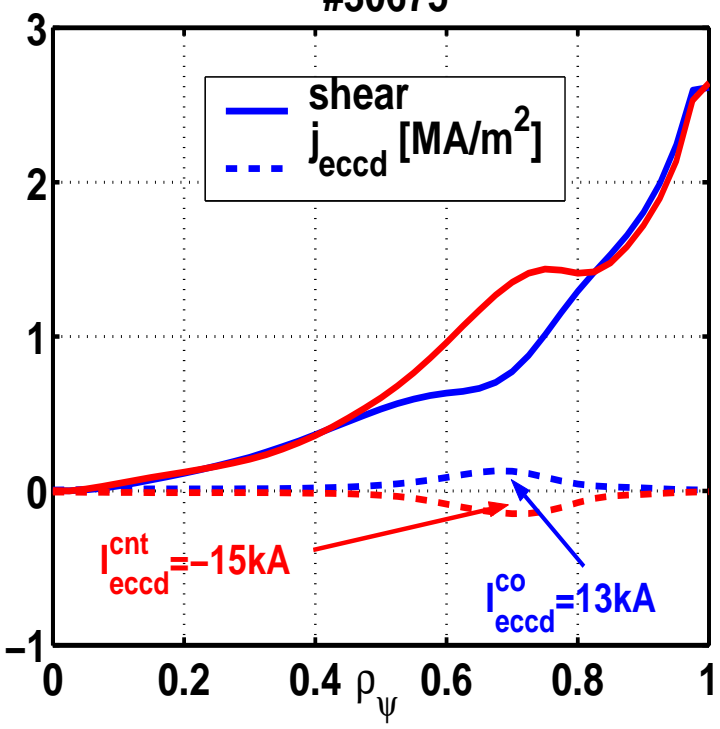

Figure 13: Simulated $q$ and $s$ profiles, and imposed $T_{\mathrm{e}}$ and $j_{\mathrm{eccd}}$ profiles of the interpretative modeling of \#30675.

\section{References}

[1] C. M. Roach et al., Nucl. Fus. 48, 125001 (2008).

[2] A. Polevoi et al., Plasma Phys. Control. Fusion 48, A449 (2006).

[3] G. T. Hoang et al., Nucl. Fus. 38, 117-132 (1998).

[4] Z. A. Pietrzyk et al., Phys. Rev. Lett. 86, 1530 (2001).

[5] O. Sauter et al., Phys. Rev. Lett. 94, 105002 (2005).

[6] A. Bottino et al., Plasma Phys. Control. Fusion 48, 215 (2006).

[7] M. A. Beer et al., Phys. Plasmas 4, 1792 (1997).

[8] C. Zucca et al., Plasma Phys. Control. Fusion 51, 015002 (2009).

[9] S. Coda et al., Nucl. Fus. 47, 714-720 (2007).

[10] S. Cirant et al., Nucl. Fusion 46, 500-511 (2006).

[11] C. Zucca, Ph.D. Thesis, EPFL, no. 4360 (2009). http://library.epfl.ch/en/theses/?nr=4360.

[12] G. V. Pereverzev et al., Max Planck - IPP Report, IPP 5/42 (1991).

[13] P. H. Rebut et al., Proc. 12th Int. Conf. on Plasma Phys. and Controlled Nuclear Fusion Research, Nice 1988, IAEA Vienna 1989, Vol 2, p. 191.

[14] R. W. Harvey and M. G. McCoy, Proc. IAEA TCM/Advances in Simulation and Modeling in Thermonuclear Plasmas, Montreal (1992).

[15] P. Nikkola et al., Nucl. Fusion 43, 1343 (2003). 
[16] C. Angioni, Ph. D. Thesis, EPFL, no. 2469 (2001). http://library.epfl.ch/en/theses/?nr=2469.

[17] J. E. Kinsey et al., Phys. Plasmas, 13, 022305 (2006).

[18] J. Candy et al., J. Comput. Phys. 186, 545 (2003). World Scientific, Borrego Springs, California, United States (1995).

[19] K. Matsuda et. al., IEEE Trans. Plasma Sci. 17, 6 (1989).

[20] O. Sauter et. al., Phys. Plasmas 6, 2834 (1999); ibidem Phys. Plasmas 9, 5140 (2002). 